TU-606

gr-qc/0011043

\title{
Diffeomorphism on Horizon as an Asymptotic Isometry of Schwarzschild Black Hole
}

\author{
M.Hotta, K.Sasaki and T.Sasaki \\ Department of Physics, Tohoku University, \\ Sendai 980-8578, Japan
}

\begin{abstract}
It is argued that the diffeomorphism on the horizontal sphere can be regarded as a nontrivial asymptotic isometry of the Schwarzschild black hole. We propose a new boundary condition of asymptotic metrics near the horizon and show that the condition admits the local time-shift and diffeomorphism on the horizon as the asymptotic symmetry.
\end{abstract}




\section{Introduction}

Recently a possibility of nontrivial asymptotic isometry near the black hole horizon has attracted much attention in the context of the microstate counting problem [1, 2, 3]. As often discussed, the no-hair theorem apparently teaches that the black hole has only three hairs; mass, angular momentum and gauge charge. Meanwhile the black hole carries enormous amount of entropy proportional to the horizon area divided by the Planck area, that is, the Bekenstein-Hawking entropy. This discrepancy, the black hole entropy problem, has annoyed a lot of theorists for long time. Among various attempts for the problem, Carlip [2] advocated recently that by virtue of the horizon structure, the "would-be gauge freedom" of general covariance supplies physical states of the Kerr black holes in arbitrary dimensions. Those states are considered as additional hairs contributing to the entropy. He argued that the Kerr black holes enjoy Virasoro horizontal isometries with non-vanishing central charge and the corresponding Bekenstein-Hawking entropies can be reproduced by state-counting via the Cardy formula in the two-dimensional conformal field theory. Unfortunately there are some shortcomings [4] in his analysis. Thus the complete resolution of the problem has not yet been achieved. However his conceptual idea has stimulated many people's interest on the analysis of the horizontal isometry.

In this paper we propose a new horizontal isometry which was never discussed so far. It is argued that the diffeomorphism on the horizon can be regarded as a nontrivial asymptotic isometry of the Schwarzschild black hole. Our boundary condition of the asymptotic metric is formulated in a general framework. and show that the asymptotic condition admits the nonvanishing charge of the diffeomorphism. This result may be significant to resolve the entropy problem. The analysis is performed for the black hole in four dimensions in this paper, but the generalization to the system in higher dimensions is straightforward. 


\section{Diffeomorphism on Horizon as Asymptotic Isometry}

In this section we propose a boundary condition of the asymptotic metrics under which the diffeomorphism on the horizon can be regarded as a subgroup of the asymptotic isometry. Let us start from the Schwarzschild metric in four dimensions.

$$
d \bar{s}^{2}=-\left(1-\frac{r_{*}}{r}\right) d t^{2}+\frac{d r^{2}}{1-\frac{r_{*}}{r}}+r^{2}\left(d \theta^{2}+\sin ^{2} \theta d \phi^{2}\right) .
$$

Here $r_{*}$ denotes the radius of the horizon. Introducing the tortoise coordinate;

$$
\rho=-\left(r-r_{*}\right)-r_{*} \ln \left[\frac{r-r_{*}}{r_{*}}\right]
$$

the metric near horizon is expressed as

$$
\begin{aligned}
d \bar{s}^{2}= & e^{-\frac{\rho}{r_{*}}}\left[1-2 e^{-\frac{\rho}{r_{*}}}+\cdots\right]\left(-d t^{2}+d \rho^{2}\right) \\
& +r_{*}^{2}\left[1+2 e^{-\frac{\rho}{r_{*}}}+\cdots\right]\left(d \theta^{2}+\sin ^{2} \theta d \phi^{2}\right) .
\end{aligned}
$$

Here the horizon at $r=r_{*}$ is mapped into $\rho=\infty$. Note that the leading term of the $t-\rho$ part of eqn(3) coincides with the Rindler metric. Thus, as known well, it shows a scaling behavior. Consider a scale transformation for the metric in eqn(3):

$$
\begin{aligned}
t^{\prime} & =\frac{\epsilon}{r_{*}} t, \\
\rho^{\prime} & =\frac{\epsilon}{r_{*}}\left[\rho+2 r_{*} \ln \left(\frac{\epsilon}{r_{*}}\right)\right],
\end{aligned}
$$

where $\epsilon$ is a constant. The transformation yields a similar asymptotic metric in which $\epsilon$ appears as its length unit in the $t-\rho$ part:

$$
\begin{aligned}
d \bar{s}^{2}= & e^{-\frac{\rho^{\prime}}{\epsilon}}\left[1-2\left(\frac{\epsilon}{r_{*}}\right)^{2} e^{-\frac{\rho^{\prime}}{\epsilon}}+\cdots\right]\left(-d t^{\prime 2}+d \rho^{\prime 2}\right) \\
& +r_{*}^{2}\left[1+2\left(\frac{\epsilon}{r_{*}}\right)^{2} e^{-\frac{\rho^{\prime}}{\epsilon}}+\cdots\right]\left(d \theta^{2}+\sin ^{2} \theta d \phi^{2}\right) .
\end{aligned}
$$


Now let us introduce the boundary condition of the asymptotic metrics which approach the background in eqn(6) and discuss the asymptotic isometry. Our philosophy to select the boundary condition is based on recent works [2, 3]. In the following argument we adopt ADM decomposition:

$$
g_{\alpha \beta}=\left[\begin{array}{cc}
-N^{2}+N_{k} N^{k} & N_{a} \\
N_{b} & h_{a b}
\end{array}\right] .
$$

Assume that the lapse and shift functions behave as

$$
\begin{aligned}
& N=e^{-\frac{\rho}{2 \epsilon}}+O\left(e^{-\frac{3 \rho}{2 \epsilon}}\right), \\
& N^{\rho}=O\left(e^{-\frac{\rho}{\epsilon}}\right), \\
& N^{\theta}=O\left(e^{-\frac{\rho}{\epsilon}}\right), \\
& N^{\phi}=O\left(e^{-\frac{\rho}{\epsilon}}\right) .
\end{aligned}
$$

Also assuming that $\xi^{\mu}=O(1)$, it is noticed due to eqns $(8) \sim(11)$ that the leading term of the surface deformation vector is fixed and does not depend on how the metric approaches the background as follows.

$$
\begin{aligned}
& \hat{\xi}^{t}=N \xi^{t} \sim e^{-\frac{\rho}{2 \epsilon}} \xi^{t}, \\
& \hat{\xi}^{a}=\xi^{a}+N^{a} \xi^{t} \sim \xi^{a} .
\end{aligned}
$$

For the spatial components of the metric the following boundary conditions are adopted.

$$
\begin{aligned}
h_{\rho \rho} & =e^{-\frac{\rho}{\epsilon}}+O\left(e^{-2 \frac{\rho}{\epsilon}}\right), \\
h_{\rho \theta} & =O\left(e^{-2 \frac{\rho}{\epsilon}}\right), \\
h_{\rho \phi} & =O\left(e^{-2 \frac{\rho}{\epsilon}}\right), \\
h_{\theta \theta} & =O(1), \\
h_{\theta \phi} & =O(1), \\
h_{\phi \phi} & =O(1) .
\end{aligned}
$$

Later we concentrate on the subset of those metrics which boundaries at $\rho=$ $\infty$ are not any physical singular surfaces. Thus the curvatures of the metrics 
are finite on the boundary even though a part of the metric components possess the superficial coordinate singularities:

$$
\begin{aligned}
& \left|R_{\mu \nu \lambda \xi} R^{\mu \nu \lambda \xi}(\rho=\infty)\right|<\infty \\
& \left|R_{\mu \nu} R^{\mu \nu}(\rho=\infty)\right|<\infty \\
& |R(\rho=\infty)|<\infty
\end{aligned}
$$

It is worth noting here that the leading behavior of the metric in eqns (8) $\sim(11)$ and (14) $\sim(16)$ preserves the horizon structure even for the asymptotic geometries, as long as the geometries are regular at $\rho=\infty$. Introducing the following global coordinates:

$$
\begin{aligned}
& T=2 \epsilon e^{-\frac{\rho}{2 \epsilon}} \sinh \left(\frac{t}{2 \epsilon}\right), \\
& X=2 \epsilon e^{-\frac{\rho}{2 \epsilon}} \cosh \left(\frac{t}{2 \epsilon}\right),
\end{aligned}
$$

the metric is reexpressed as

$$
\begin{aligned}
d s^{2}= & -d T^{2}+d X^{2} \\
& +O(X-T)(d X+d T) d \theta+O(X+T)(d X-d T) d \phi \\
& +O\left((X-T)^{2}\right)(d X+d T)^{2} \\
& +O((X+T)(X-T))(d X+d T)(d X-d T) \\
& +O\left((X+T)^{2}\right)(d X-d T)^{2} \\
& +O(1) d \theta^{2}+O(1) d \theta d \phi+O(1) d \phi^{2} .
\end{aligned}
$$

In this coordinate the boundary at $\rho=\infty$ is mapped into $\pm T=X>0$. Clearly the fall-off condition in eqn(25) guarantees that worldlines with which the boundary is completely covered:

$$
\begin{aligned}
& T=X>0(\text { or }-T=X>0), \\
& \theta=\text { const } \\
& \phi=\text { const }
\end{aligned}
$$

are exactly null geodesics for every asymptotic metric. Therefore the horizontal structure is really exposed, that is, any physical object across the boundary $(\rho=\infty)$ cannot come back to the outside region $(\rho<\infty)$. 
Strictly speaking, we call the boundary "horizon" just in the sense of the Rindler wedge. The wedge structure has often been argued to be very essential for the black hole thermodynamics from the early stage of investigation on the Hawking radiation [5]. This is mainly because the thermal noise of quantum fields appears in the wedge(Rindler) coordinates. We believe that our boundary condition which involves the wedge structure is rather natural to apply to the black hole entropy problem.

So far we have mentioned a horizontal interpretation of our boundary in a rather geometrical way by introducing the boundary null geodesics. If more coordinate-independent expression of our fall-off metric condition near the horizon can be attained, it will help us understand more clearly the geometrical meaning of the boundary condition. We think that it is a significant open problem.

In our formulation we can analyze simultaneously the Schwarzschild black holes with various radii larger than $\epsilon$. By choosing the coordinates adequately, the metrics of the black holes belong to the same class of asymptotic equivalence. Also stress that the constant $\epsilon$ is able to be selected arbitrarily in the classical gravity. For each value of $\epsilon$, one can always span a coordinate frame in which the leading terms of the metrics of the black holes with $r_{*}>\epsilon$ are given by eqn(6). If it is possible to observe the metric deviations near the horizon, it may be useful for the analysis to fix the constant $\epsilon$ of order of the device scale. On the other hand, in the microstate counting of the black hole, it might be crucial to choose the Planck length for the constant $\epsilon$.

The asymptotic behaviors of the inverse spatial metric is obtained straightforwardly from eqns (14) $\sim(19)$ as follows.

$$
\left[h^{a b}\right]=\left[\begin{array}{ccc}
e^{\frac{\rho}{\epsilon}}+O(1) & O\left(e^{-\frac{\rho}{\epsilon}}\right) & O\left(e^{-\frac{\rho}{\epsilon}}\right) \\
O\left(e^{-\frac{\rho}{\epsilon}}\right. & O(1) & O(1) \\
O\left(e^{-\frac{\rho}{\epsilon}}\right. & O(1) & O(1)
\end{array}\right] .
$$

Note that the leading term of $h^{\rho \rho}$ always coincides with the background term; $e^{\frac{\rho}{\epsilon}}$. The boundary conditions of the connections are also easily found using eqns (14) $\sim(29)$.

$$
\begin{aligned}
& \Gamma_{\rho \rho}^{(3) \rho}=O(1), \\
& \Gamma_{\rho \rho}^{(3) \alpha}=O\left(e^{-2 \frac{\rho}{\epsilon}}\right),
\end{aligned}
$$




$$
\begin{aligned}
& \Gamma_{\alpha \rho}^{(3) \rho}=O\left(e^{-\frac{\rho}{\epsilon}}\right), \\
& \Gamma_{\alpha \beta}^{(3) \rho}=O(1), \\
& \Gamma_{\rho \beta}^{(3) \alpha}=O\left(e^{-\frac{\rho}{\epsilon}}\right), \\
& \Gamma_{\alpha \beta}^{(3) \gamma}=O(1),
\end{aligned}
$$

where $\alpha, \beta$ and $\gamma$ take the angular indices $\theta$ and $\phi$. Under the conditions in eqns(8) $\sim(11)$ and $(14) \sim(19)$, it is checked that the extrinsic curvature;

$$
K_{a b}=\frac{1}{2 N}\left(N_{a \mid b}+N_{b \mid a}-\partial_{t} h_{a b}\right)
$$

behaves near the horizon as

$$
\begin{aligned}
& K_{\rho \rho}=O\left(e^{-\frac{3 \rho}{2 \epsilon}}\right), \\
& K_{\rho \theta}=O\left(e^{-\frac{\rho}{2 \epsilon}}\right), \\
& K_{\rho \phi}=O\left(e^{-\frac{\rho}{2 \epsilon}}\right), \\
& K_{\theta \theta}=O\left(e^{\frac{\rho}{2 \epsilon}}\right), \\
& K_{\theta \phi}=O\left(e^{\frac{\rho}{2 \epsilon}}\right), \\
& K_{\phi \phi}=O\left(e^{\frac{\rho}{2 \epsilon}}\right) .
\end{aligned}
$$

Here it has been assumed that $\partial_{t} h_{a b}$ shows the same asymptotic behavior of $h_{a b}$ near the horizon, except $h_{\rho \rho}$. For the $(\rho \rho)$ component, we assume from eqn(14) that $\partial_{t} h_{\rho \rho}=O\left(e^{-2 \frac{\rho}{\epsilon}}\right)$ holds. As usual, the canonical momentum tensor is defined as

$$
\Pi^{a b}=\frac{\sqrt{h}}{16 \pi G}\left[K h^{a b}-K^{a b}\right],
$$

and behaves under the condition near the horizon as

$$
\begin{aligned}
& \Pi^{\rho \rho}=O\left(e^{\frac{\rho}{\epsilon}}\right), \\
& \Pi^{\rho \alpha}=O(1), \\
& \Pi^{\alpha \beta}=O(1) .
\end{aligned}
$$

It is quite significant to emphasize that the following transformation possesses the well-defined charges and can be regarded as a subgroup of the 
asymptotic isometry.

$$
\begin{aligned}
& \xi^{t}=T(\theta, \phi)+O\left(e^{-\frac{\rho}{\epsilon}}\right) \\
& \xi^{\rho}=0 \\
& \xi^{\theta}=V^{\theta}(\theta, \phi)+O\left(e^{-2 \frac{\rho}{\epsilon}}\right) \\
& \xi^{\phi}=V^{\phi}(\theta, \phi)+O\left(e^{-2 \frac{\rho}{\epsilon}}\right)
\end{aligned}
$$

where $T$ and $V^{\alpha}$ are regular scalar and vector functions on the horizontal sphere. The vector part $V^{\alpha}$ consists of the the diffeomorphism on the sphere.

Let us examine the canonical charges explicitly. The canonical Hamiltonian and momentum densities are written down as

$$
\begin{aligned}
& \mathcal{H}_{t}=\frac{16 \pi G}{\sqrt{h}}\left[\Pi^{a b} \Pi_{a b}-\frac{1}{2} \Pi^{2}\right]-\frac{\sqrt{h}}{16 \pi G} R^{(3)}+\mathcal{H}_{t}^{\text {matter }}, \\
& \mathcal{H}_{a}=-\left.2 \Pi_{a b}\right|^{b}+\mathcal{H}_{a}^{\text {matter }} .
\end{aligned}
$$

where $G$ is the gravitational constant, $R^{(3)}$ is the scalar curvature of the spatial section and $\mathcal{H}_{\mu}^{\text {matter }}$ represents matter contribution of the model if it exists. Then the bulk part of the canonical generator is expressed using eqns (51) and (52) as

$$
H[\hat{\xi}]=\int d^{3} x\left[\hat{\xi}^{t} \mathcal{H}_{t}+\hat{\xi}^{a} \mathcal{H}_{a}\right]
$$

Taking variations of $H$ with respect to $h_{a b}$ and $\Pi^{a b}$ yields

$$
\delta H[\hat{\xi}]=\int d^{3} x\left(\partial_{t} \Pi^{a b} \delta h_{a b}-\partial_{t} h_{a b} \delta \Pi^{a b}\right)-\delta Q,
$$

where the equations of motion have been used to get the time derivative terms and

$$
\begin{gathered}
\delta Q=\int d \phi d \theta\left[\frac{1}{16 \pi G} \sqrt{h}\left(h^{a c} h^{b \rho}-h^{a b} h^{c \rho}\right)\left(\hat{\xi}^{t} \delta h_{a b \mid c}-\hat{\xi}_{\mid c}^{t} \delta h_{a b}\right)\right. \\
\left.+2 \hat{\xi}^{a} \delta \Pi_{a}^{\rho}-\hat{\xi}^{\rho} \Pi^{a b} \delta h_{a b}\right]_{\rho=\infty} .
\end{gathered}
$$

If $\delta Q$ in eqn (55) can be integrated rigorously, the canonical charge of the surface deformation is defined by $Q=\int \delta Q$. Interestingly, under the conditions in eqns $(\sqrt{12}) \sim(19)$ and $(44) \sim(46)$ it is proven that the integration of $\delta Q$ for 
the transformation in eqns $(47) \sim(50)$ is really possible. The non-vanishing contribution in the limit of $\rho \rightarrow \infty$ turns out to be just two terms as follows.

$$
\delta Q=\int d \phi d \theta\left[-\frac{1}{32 \pi G \epsilon} \sqrt{\operatorname{det}\left[h_{\alpha \beta}\right]} \xi^{t} h^{\alpha \beta} \delta h_{\alpha \beta}+2 \xi^{\alpha} \delta \Pi_{\alpha}^{\rho}\right]_{\rho=\infty} .
$$

Here $\alpha$ and $\beta$ run just in the angular part $\theta$ and $\phi$. Due to the relation:

$$
\delta \sqrt{\operatorname{det}\left[h_{\alpha \beta}\right]}=\frac{1}{2} \sqrt{\operatorname{det}\left[h_{\alpha \beta}\right]} h^{\alpha \beta} \delta h_{\alpha \beta},
$$

the integration results in

$$
Q=\int d \phi d \theta\left[-\frac{1}{16 \pi G \epsilon} \xi^{t} \sqrt{\operatorname{det}\left[h_{\alpha \beta}\right]}+2 \xi^{\alpha} \Pi_{\alpha}^{\rho}\right]_{\rho=\infty} .
$$

Both two right-hand-side terms in eqn (58) can be shown finite under the asymptotic conditions. Also we are able to rewrite the expression of $Q$ by use of the original surface deformation vector $\hat{\xi}^{\mu}$ as follows.

$$
Q=\int d \phi d \theta\left[\frac{\sqrt{h}}{8 \pi G} h^{\rho \rho} \partial_{\rho} \hat{\xi}^{t}+2 \hat{\xi}^{\alpha} \Pi_{\alpha}^{\rho}\right]_{\rho=\infty},
$$

where we have used the following asymptotic forms:

$$
\begin{aligned}
& \sqrt{h} \sim e^{-\frac{\rho}{2 \epsilon}} \sqrt{\operatorname{det}\left[h_{\alpha \beta}\right]}, \\
& h^{\rho \rho} \sim e^{\frac{\rho}{\epsilon}}, \\
& N \sim e^{-\frac{\rho}{2 \epsilon}} .
\end{aligned}
$$

In Appendix it is commented that the canonical charge in eqn (58) coincides explicitly with the Noether charge of the transformation in eqns $(47) \sim(50)$.

In the next section we calculate the charges of black hole solutions and analyze the representations.

\section{Horizontal Charge of Black Hole Solutions}


In this section we evaluate the horizontal charges of the black hole solutions. For convenience of later explanation, let us first introduce an angular metric on a unit sphere as follows.

$$
\begin{aligned}
d \Omega^{2} & =\sigma_{\theta \theta} d \theta^{2}+2 \sigma_{\theta \phi} d \theta d \phi+\sigma_{\phi \phi} d \phi^{2} \\
& =d \theta^{2}+\sin ^{2} \theta d \phi^{2} .
\end{aligned}
$$

In general it is possible to decompose the regular vector field $V^{\alpha}$ in eqns(49) and (50) into sum of a divergenceless (or area preserving) part and a rotationless part as

$$
V^{\alpha}=-\frac{1}{\sqrt{\sigma}} \epsilon^{\alpha \beta} \nabla_{\beta}^{(2)} \Psi_{1}(\theta, \phi)+\nabla^{(2) \alpha} \Psi_{2}(\theta, \phi),
$$

where $\Psi_{k}$ is a regular scalar function on the sphere, $\nabla^{(2)}$ is the covariant derivative associated with the metric in eqn (60) and

$$
\begin{aligned}
& \epsilon^{\theta \phi}=-\epsilon^{\phi \theta}=1, \\
& \epsilon^{\theta \theta}=\epsilon^{\phi \phi}=0, \\
& \sqrt{\sigma}=\sin \theta .
\end{aligned}
$$

Now we have three types of the generators of the transformations in eqns (47) $\sim(50) ; T, \Psi_{1}$ and $\Psi_{2}$ and can expand them using the spherical harmonic functions as follows.

$$
\begin{aligned}
& T=\sum_{l m} a_{l m} Y_{l m}(\theta, \phi), \\
& \Psi_{k}=\sum_{l m} b_{l m}^{(k)} Y_{l m}(\theta, \phi) .
\end{aligned}
$$

Consequently all the independent generators of the symmetry can be listed as

$$
\begin{aligned}
& J_{l m}^{(t)}=Y_{l m} \partial_{t}+O\left(e^{-\frac{\rho}{\epsilon}}\right), \\
& J_{l m}^{(1)}=\frac{1}{\sin \theta}\left(\partial_{\theta} Y_{l m} \partial_{\phi}-\partial_{\phi} Y_{l m} \partial_{\theta}\right)+O\left(e^{-2 \frac{\rho}{\epsilon}}\right), \\
& J_{l m}^{(2)}=\partial_{\theta} Y_{l m} \partial_{\theta}+\frac{1}{\sin ^{2} \theta} \partial_{\phi} Y_{l m} \partial_{\phi}+O\left(e^{-2 \frac{\rho}{\epsilon}}\right),
\end{aligned}
$$


where $J^{(t)}$ generates the local time-shift on the horizon. The charges $J^{(1)}$ and $J^{(2)}$ generate the two-dimensional diffeomorphism on the sphere. In order to analyze the commutation relations of the generators, it is useful to define three structure coefficients $G_{l m l^{\prime} m^{\prime}}^{l^{\prime \prime} m^{\prime \prime}}, C_{l m l^{\prime} m^{\prime}}^{l^{\prime \prime} m^{\prime \prime}}$ and $D_{l m l^{\prime} m^{\prime}}^{l^{\prime \prime} m^{\prime \prime}}$ as

$$
\begin{aligned}
& Y_{l m} Y_{l^{\prime} m^{\prime}}=\sum G_{l m l^{\prime} m^{\prime}}^{l^{\prime \prime} m_{l^{\prime \prime} m^{\prime \prime}}}, \\
& \frac{1}{\sin \theta}\left(\partial_{\theta} Y_{l m} \partial_{\phi} Y_{l^{\prime} m^{\prime}}-\partial_{\phi} Y_{l m} \partial_{\theta} Y_{l^{\prime} m^{\prime}}\right)=\sum C_{l m l^{\prime} m^{\prime}}^{l^{\prime \prime} m^{\prime \prime} Y^{\prime \prime}}, \\
& \partial_{\theta} Y_{l m} \partial_{\theta} Y_{l^{\prime} m^{\prime}}+\frac{1}{\sin ^{2} \theta} \partial_{\phi} Y_{l m} \partial_{\phi} Y_{l^{\prime} m^{\prime}}=\sum D_{l m l^{\prime} m^{\prime}}^{l^{\prime \prime} m^{\prime \prime}} Y_{l^{\prime \prime} m^{\prime \prime}}
\end{aligned}
$$

By definition the coefficients satisfy the following permutation relations.

$$
\begin{aligned}
& G_{l^{\prime} m^{\prime} l m}^{l^{\prime \prime} m^{\prime \prime}}=G_{l m l^{\prime} m^{\prime}}^{l^{\prime \prime} m^{\prime \prime}} \\
& C_{l^{\prime} m^{\prime} l m}^{l^{\prime \prime} m^{\prime \prime}}=-C_{l m l^{\prime} m^{\prime}}^{l^{\prime \prime} m^{\prime \prime}} \\
& D_{l^{\prime} m^{\prime} l m}^{l^{\prime \prime} m^{\prime \prime}}=D_{l m l^{\prime} m^{\prime}}^{l^{\prime \prime} m^{\prime \prime}}
\end{aligned}
$$

Also the following relations are easily proven from the definitions.

$$
\begin{aligned}
& G_{l m l^{\prime} m^{\prime}}^{00}=\frac{1}{\sqrt{4 \pi}} \delta_{l l^{\prime}} \delta_{m m^{\prime}}, \\
& C_{l m l^{\prime} m^{\prime}}^{00}=0 \\
& D_{l m l^{\prime} m^{\prime}}^{00}=\frac{l(l+1)}{\sqrt{4 \pi}} \delta_{l l^{\prime}} \delta_{m m^{\prime}} .
\end{aligned}
$$

Then all the commutators can be expressed by use of the coefficients.

$$
\begin{aligned}
& {\left[J_{l m}^{(t)}, J_{l^{\prime} m^{\prime}}^{(t)}\right]=0,} \\
& {\left[J_{l m}^{(1)}, J_{l^{\prime} m^{\prime}}^{(t)}\right]=\sum C_{l m l^{\prime} m^{\prime}}^{l^{\prime \prime} m_{l^{\prime \prime} m^{\prime \prime}}^{\prime \prime}},(t)} \\
& {\left[J_{l m}^{(2)}, J_{l^{\prime} m^{\prime}}^{(t)}\right]=\sum D_{l m l^{\prime} m^{\prime}}^{l^{\prime \prime} m^{\prime \prime}} J_{l^{\prime \prime} m^{\prime \prime}}^{(t)},} \\
& {\left[J_{l m}^{(1)}, J_{l^{\prime} m^{\prime}}^{(1)}\right]=\sum C_{l m l^{\prime} m^{\prime}}^{l^{\prime \prime} m^{\prime \prime}} J_{l^{\prime \prime} m^{\prime \prime}}^{(1)},} \\
& {\left[J_{l m}^{(2)}, J_{l^{\prime} m^{\prime}}^{(2)}\right]=\sum C_{l m l^{\prime} m^{\prime}}^{l^{\prime \prime} m^{\prime \prime}} J_{l^{\prime \prime} m^{\prime \prime}}^{(1)},} \\
& {\left[J_{l m}^{(1)}, J_{l^{\prime} m^{\prime}}^{(2)}\right]}
\end{aligned}
$$




$$
\begin{aligned}
= & \sum_{l^{\prime \prime} \neq 0}\left[D_{l m l^{\prime} m^{\prime}}^{l^{\prime \prime} m^{\prime \prime}} \frac{l^{\prime}\left(l^{\prime}+1\right)-l^{\prime \prime}\left(l^{\prime \prime}+1\right)}{l^{\prime \prime}\left(l^{\prime \prime}+1\right)}-G_{l m l^{\prime} m^{\prime}}^{l^{\prime \prime} m^{\prime \prime}} \frac{l(l+1) l^{\prime}\left(l^{\prime}+1\right)}{l^{\prime \prime}\left(l^{\prime \prime}+1\right)}\right] J_{l^{\prime \prime} m^{\prime \prime}}^{(1)} \\
& +\sum_{l^{\prime \prime} \neq 0} C_{l m l^{\prime} m^{\prime}}^{l^{\prime \prime} m^{\prime \prime}} \frac{l^{\prime}\left(l^{\prime}+1\right)}{l^{\prime \prime}\left(l^{\prime \prime}+1\right)} J_{l^{\prime \prime} m^{\prime \prime}}^{(2)} .
\end{aligned}
$$

It is noticed here that the Cartan subalgebra of the symmetry consists of $J_{l m}^{(t)}$. All of generators $J_{l m}^{(1)}$ do not commute with $J_{l^{\prime} m^{\prime}}^{(t)}$ except when $l=l^{\prime}$ and $m=m^{\prime}$ (or $l^{\prime}=0$ and $\left.m^{\prime}=0\right)$. Also $J_{l m}^{(2)}$ do not commute with $J_{l^{\prime} m^{\prime}}^{(t)}$ except when $l^{\prime}=0$ and $m^{\prime}=0$.

Substituting eqns $(47) \sim(50)$ and the black hole solution (eqn (6)) into eqn (58) yields

$$
Q\left[T, V^{\alpha}\right]=-\frac{r_{*}^{2}}{16 \pi G \epsilon} \int d \phi d \theta \sin \theta T(\theta, \phi) .
$$

Therefore, using the decomposition in eqns (65) and (66), we get each component of the charges as follows.

$$
\begin{gathered}
Q_{00}^{(t)}=-\frac{\sqrt{4 \pi} r_{*}^{2}}{16 \pi G \epsilon}, \\
Q_{l m}^{(t)}=0, \quad(l \geq 1) \\
Q_{l m}^{(1)}=0, \\
Q_{l m}^{(2)}=0 .
\end{gathered}
$$

It is also possible to verify that when the background is infinitesimally transformed by $T_{1}$ and $V_{1}^{\alpha}$, the charge $Q_{2}=Q\left[T_{2}, V_{2}^{\alpha}\right]$ changes in the usual way and any anomalous term like central extension is not needed:

$$
\delta_{1} Q_{2}=\frac{r_{*}^{2}}{16 \pi G \epsilon} \int d \phi d \theta \sin \theta\left[V_{1}^{\alpha} \partial_{\alpha} T_{2}-V_{2}^{\alpha} \partial_{\alpha} T_{1}\right]
$$

It is easy to construct the irreducible representation of the symmetry for the metrics of the black hole solutions with radius $r_{*}$. The transformation of the background in eqn (6) by

$$
\begin{aligned}
& t^{\prime}=t+P(\theta, \phi)+O\left(e^{-\frac{\rho}{\epsilon}}\right), \\
& \rho^{\prime}=\rho, \\
& \theta^{\prime}=F(\theta, \phi)+O\left(e^{-2 \frac{\rho}{\epsilon}}\right), \\
& \phi^{\prime}=G(\theta, \phi)+O\left(e^{-2 \frac{\rho}{\epsilon}}\right)
\end{aligned}
$$


yields all of the asymptotic metrics in the representation. Here the regular function $P$ is an arbitrary local time-shift on the horizontal sphere. The functions $F$ and $G$ define an arbitrary regular coordinate transformation on the sphere. It turns out that any asymptotic metric in the representation takes the following form.

$$
\begin{aligned}
d s^{2}= & e^{-\frac{\rho}{\epsilon}}\left(-d t^{2}+d \rho^{2}\right) \\
& -2 e^{-\frac{\rho}{\epsilon}}\left[\partial_{\theta} P d \theta+\partial_{\phi} P d \phi\right] d t \\
& +r_{*}^{2}\left[\left(\partial_{\theta} F\right)^{2}+\sin ^{2} F\left(\partial_{\theta} G\right)^{2}\right] d \theta^{2} \\
& +2 r_{*}^{2}\left[\partial_{\theta} F \partial_{\phi} F+\sin ^{2} F \partial_{\theta} G \partial_{\phi} G\right] d \theta d \phi \\
& +r_{*}^{2}\left[\left(\partial_{\phi} F\right)^{2}+\sin ^{2} F\left(\partial_{\phi} G\right)^{2}\right] d \phi^{2} \\
& +\ldots
\end{aligned}
$$

where $+\cdots$ denotes terms which do not contribute to the charges of the symmetry. For the metric in eqn (95), the charges are evaluated as

$$
Q\left[T, V^{\alpha}\right]=-\frac{r_{*}^{2}}{16 \pi G \epsilon} \int d \phi d \theta \sin F\left[\partial_{\theta} F \partial_{\phi} G-\partial_{\phi} F \partial_{\theta} G\right]\left(T+V^{\alpha} \partial_{\alpha} P\right)
$$

and the representation has been really proven to be non-singlet.

From the viewpoint of the black hole thermodynamics, asymptotic states which charges of the Cartan subalgebra take the same values of the background may be worth analyzing in detail. This is because degeneracy of the eigenstates may supply "hair degrees of freedom" which contributes to the Bekenstein-Hawking entropy, as many people have already pointed out [2, 3]. However, opposed to the attempts with central extended Virasoro symmetries, it seems fairly difficult to perform microstate counting because of a lack of clear understanding of the quantum gravity. Thus let us just comment on the classical charges probably corresponding to the coherent states of the Cartan subalgebra. It can be noticed that the metrics in eqn(95) which $Q_{l m}^{(t)}$ are given by eqns(86) and (87) must satisfy

$$
\sin F\left[\partial_{\theta} F \partial_{\phi} G-\partial_{\phi} F \partial_{\theta} G\right]=\sin \theta,
$$

that is, $F$ and $G$ in eqns(93) and (94) must generate the area-preserving diffeomorphism on the sphere. The diffeomorphism charges of the metrics satisfying eqn(97) are evaluated as follows.

$$
Q_{l m}^{(1)}=0,
$$




$$
Q_{l m}^{(2)}=-\frac{r_{*}^{2}}{16 \pi G \epsilon} l(l+1) \int d \theta d \phi \sin \theta Y_{l m}(\theta, \phi) P(\theta, \phi)
$$

Thus the divergenceless (area-preserving) part of the charges vanishes and only the rotationless part is nontrivial.

\section{Summary}

In this paper it has been reported that the general coordinate transformation on the horizon in eqns (47) $\sim(50)$ must be treated as not a gauge freedom but a subgroup of the asymptotic isometry. Firstly, taking account of the near-horizon form of the Schwarzschild solution in eqn(6), we have proposed a rather general condition of the asymptotic metrics in eqns (8) (11) and $(\sqrt{14}) \sim(\sqrt{19})$. Then it has been proven that the non-vanishing charge of the symmetry really appears, as seen in eqn(96). Therefore we conclude that the "would-be gauge freedom" in eqns (47) (50) cannot be gauged away and thus might be relevant for the black hole entropy counting.

\section{APPENDIX}

In this appendix we show explicitly that the canonical charge $Q$ in eqn (58) is really the Noether charge for the transformation in eqns $(47) \sim(50)$. Let us first consider the covariant Lagrangian density for a scalar matter field as an example and the gravity as follows.

$$
\sqrt{-g} \mathcal{L}_{o}=\frac{\sqrt{-g}}{16 \pi G} R+\sqrt{-g} \mathcal{L}_{\text {matter }}\left(\phi, \partial \phi, g^{\alpha \beta}\right)
$$

where $\mathcal{L}_{\text {matter }}$ denotes the contribution of the scalar field. The equations of 
motion simply read

$$
\begin{aligned}
& \frac{\partial \sqrt{-g} \mathcal{L}_{o}}{\partial g_{\alpha \beta}}-\partial_{\mu} \frac{\partial \sqrt{-g} \mathcal{L}_{o}}{\partial \partial_{\mu} g_{\alpha \beta}}+\partial_{\mu} \partial_{\nu} \frac{\partial \sqrt{-g} \mathcal{L}_{o}}{\partial \partial_{\mu} \partial_{\nu} g_{\alpha \beta}}=0, \\
& \frac{\partial \sqrt{-g} \mathcal{L}_{o}}{\partial \phi}-\partial_{\mu} \frac{\partial \sqrt{-g} \mathcal{L}_{o}}{\partial \partial_{\mu} \phi}=0 .
\end{aligned}
$$

Next let us discuss the Lie derivative with respect to a vector field $\xi^{\mu}$. The Lie derivatives of the metric and the scalar field are given as

$$
\begin{aligned}
& \delta_{\xi} g_{\alpha \beta}=\nabla_{\alpha} \xi_{\beta}+\nabla_{\beta} \xi_{\alpha}, \\
& \delta_{\xi} \phi=\xi^{\alpha} \nabla_{\alpha} \phi .
\end{aligned}
$$

Covariance of the Lagrangian density makes its Lie derivative form simple as

$$
\delta_{\xi}\left(\sqrt{-g} \mathcal{L}_{o}\right)=\partial_{\mu}\left(\xi^{\mu} \sqrt{-g} \mathcal{L}_{o}\right) .
$$

By virtue of the Noether theorem, it is known that the equation of motion ensures the existence of conserved current :

$$
\partial_{\mu} \bar{J}^{\mu}=0
$$

where the current is defined as

$$
\begin{aligned}
\bar{J}^{\mu}= & {\left[\frac{\partial \sqrt{-g} \mathcal{L}_{o}}{\partial \partial_{\mu} g_{\alpha \beta}}-\partial_{\nu} \frac{\partial \sqrt{-g} \mathcal{L}_{o}}{\partial \partial_{\mu} \partial_{\nu} g_{\alpha \beta}}\right] \delta_{\xi} g_{\alpha \beta}+\frac{\partial \sqrt{-g} \mathcal{L}_{o}}{\partial \partial_{\mu} \partial_{\nu} g_{\alpha \beta}} \partial_{\nu} \delta_{\xi} g_{\alpha \beta}-\xi^{\mu} \sqrt{-g} \mathcal{L}_{o} } \\
& +\frac{\partial \sqrt{-g} \mathcal{L}_{o}}{\partial \partial_{\mu} \phi} \delta_{\xi} \phi
\end{aligned}
$$

The spatial volume integration of $\bar{J}^{0}$ gives the Noether charge of general covariance of the Lagrangian density.

To incorporate the canonical Regge-Teitelboim action:

$$
\begin{aligned}
\int d^{4} x \sqrt{-g} \mathcal{L}= & \int d^{4} x\left[\Pi^{a b} \partial_{t} h_{a b}-N \mathcal{H}_{t}-N^{a} \mathcal{H}_{a}+\sqrt{-g} \mathcal{L}_{\text {matter }}\right] \\
& -\int d t Q\left[\xi^{t}=1, \xi^{a}=0\right]
\end{aligned}
$$

into the consideration, we next subtract a total derivative term from the Lagrangian density as follows.

$$
\sqrt{-g} \mathcal{L}=\sqrt{-g} \mathcal{L}_{o}-\partial_{\mu} D^{\mu}\left(g_{\alpha \beta}, \partial_{\gamma} g_{\alpha \beta}\right) .
$$


where $D^{\mu}$ in the derivative term is fixed for the horizontal boundary as

$$
\begin{aligned}
D^{0} & =-\frac{\sqrt{h}}{8 \pi G} K, \\
D^{\rho} & =\frac{\sqrt{h}}{8 \pi G}\left[K^{\rho b} N_{b}-N^{\mid \rho}\right]-\frac{1}{16 \pi G \epsilon} \sqrt{\operatorname{det}\left[h_{\alpha \beta}\right]}, \\
D^{\alpha} & =\frac{\sqrt{h}}{8 \pi G}\left[K^{\alpha b} N_{b}-N^{\mid \alpha}\right] .
\end{aligned}
$$

In this case the Lie derivative of the Lagrangian density is modified by the total divergent term as

$$
\delta_{\xi} \sqrt{-g} \mathcal{L}=\partial_{\mu} \Lambda^{\mu}
$$

where

$$
\Lambda^{\mu}=\xi^{\mu} \sqrt{-g} \mathcal{L}+\xi^{\mu} \partial_{\nu} D^{\nu}-\delta_{\xi} D^{\mu} .
$$

and eqn (113) yields a new current conservation law as follows.

$$
\partial_{\mu} J^{\mu}=0,
$$

where the current is re-defined as

$$
\begin{aligned}
J^{\mu}= & {\left[\frac{\partial \sqrt{-g} \mathcal{L}}{\partial \partial_{\mu} g_{\alpha \beta}}-\partial_{\nu} \frac{\partial \sqrt{-g} \mathcal{L}}{\partial \partial_{\mu} \partial_{\nu} g_{\alpha \beta}}\right] \delta_{\xi} g_{\alpha \beta}+\frac{\partial \sqrt{-g} \mathcal{L}}{\partial \partial_{\mu} \partial_{\nu} g_{\alpha \beta}} \partial_{\nu} \delta_{\xi} g_{\alpha \beta}-\Lambda^{\mu} } \\
& +\frac{\partial \sqrt{-g} \mathcal{L}}{\partial \partial_{\mu} \phi} \delta_{\xi} \phi
\end{aligned}
$$

Let us define coefficients $B_{\nu}^{\mu}, C_{\nu}^{\mu \alpha}$ and $F_{\nu}^{\mu \alpha \beta}$ as

$$
J^{\mu}=B_{\nu}^{\mu} \xi^{\nu}+C_{\nu}^{\mu \alpha} \partial_{\alpha} \xi^{\nu}+F_{\nu}^{\mu \alpha \beta} \partial_{\alpha} \partial_{\beta} \xi^{\nu} .
$$

Then the Noether theorem tells that the following four relations must hold simultaneously.

$$
\begin{aligned}
& \partial_{\mu} B_{\nu}^{\mu}=0, \\
& B_{\nu}^{\mu}+\partial_{\alpha} C_{\nu}^{\alpha \mu}=0, \\
& \frac{1}{2}\left(C_{\nu}^{\alpha \beta}+C_{\nu}^{\beta \alpha}\right)+\partial_{\mu} F_{\nu}^{\mu \alpha \beta}=0, \\
& F_{\nu}^{\alpha \beta \gamma}+F_{\nu}^{\beta \gamma \alpha}+F_{\nu}^{\gamma \alpha \beta}=0 .
\end{aligned}
$$


Using the above relations, the charge density $J^{0}$ can be reexpressed by a spatial total derivative term as follows.

$$
J^{0}=\partial_{a}\left[\partial_{0}\left(F_{\mu}^{a 00} \xi^{\mu}\right)-C_{\mu}^{a 0} \xi^{\mu}-2 F_{\mu}^{a 0 \nu} \partial_{\nu} \xi^{\mu}\right] .
$$

Substituting the asymptotic conditions $(12) \sim(19)$ and $(44) \sim(46)$, it can be shown that the Noether charge really equals to the canonical charge $Q$ as follows.

$$
\begin{aligned}
Q_{\text {Noether }} & =\int d \rho d \theta d \phi J^{0} \\
& =-\int d \theta d \phi\left[C_{0}^{\rho 0} \xi^{t}+C_{\alpha}^{\rho 0} \xi^{\alpha}\right]_{\rho=\infty} \\
& =\int d \theta d \phi\left[-\frac{1}{16 \pi G \epsilon} \xi^{t} \sqrt{\operatorname{det}\left[h_{\alpha \beta}\right]}+2 \xi^{\alpha} \Pi_{\alpha}^{\rho}\right]_{\rho=\infty}=Q[\xi] .
\end{aligned}
$$




\section{References}

[1] S. Carlip,Phys.Rev.D51, 632(1995).

A.P.Balachandran, L.Chandar and A.Momen, Nucl. Phys. B461,581(1996).

A. Ashtekar, J. Baez, A. Corichi and K. Krasnov, Phys.Rev.Lett.80,904(1998).

[2] S. Carlip, Phys.Rev.Lett.82,2828(1999).

[3] S.N. Solodukhin, Phys.Lett.B454,213(1999).

Feng-Li Lin and Yong-Shi Wu, Phys.Lett.B453,222(1999).

S. Carlip,Class.Quant.Grav.16,3327(1999).

Jiliang Jing and Mu-Lin Yan, Phys.Rev.D62,104013(2000):

Statistical Entropy of a Stationary Dilaton Black Hole from Cardy Formula,gr-qc/0005105.

S. Das, A. Ghosh and P. Mitra, Phys.Rev.63, 024023(2001).

R. Brustein, Causal Boundary Entropy From Horizon Conformal Field Theory, hep-th/0005266.

[4] Mu-In Park and Jeongwon Ho, Phys.Rev.Lett.83,5595 (1999).

V.O. Soloviev,Phys.Rev.D61,027502(2000).

S. Carlip, Phys.Rev.Lett.83,5596(1999).

[5] See N.D.Birrell and P.C.W.Davies, Quantum Fields in Curved Space (1982) Cambridge University Press. 